\title{
Nitrogen dissociation degree in the diffusion region of a helicon plasma source obtained by atomic lines to molecular band intensities ratio
}

Costel Biloiu

Earl E. Scime

Ioana A. Biloiu

Xuan Sun

Follow this and additional works at: https://researchrepository.wvu.edu/faculty_publications

\section{Digital Commons Citation}

Biloiu, Costel; Scime, Earl E.; Biloiu, Ioana A.; and Sun, Xuan, "Nitrogen dissociation degree in the diffusion region of a helicon plasma source obtained by atomic lines to molecular band intensities ratio" (2007). Faculty Scholarship. 243.

https://researchrepository.wvu.edu/faculty_publications/243 


\title{
Nitrogen dissociation degree in the diffusion region of a helicon plasma source obtained by atomic lines to molecular band intensities ratio
}

\author{
Costel Biloiu ${ }^{a)}$ \\ Varian Semiconductor Equipment Associates, 35 Dory Road, Gloucester, Massachusetts 01930, USA \\ Earl E. Scime and loana A. Biloiu \\ Physics Department, West Virginia University, Morgantown, West Virginia 26506, USA \\ Xuan Sun \\ Los Alamos National Laboratory, Los Alamos, New Mexico 87545, USA
}

(Received 2 July 2007; accepted 20 July 2007; published online 14 September 2007)

\begin{abstract}
Estimates of the dissociation degree in the diffusion region of a nitrogen helicon plasma source based on optical emission spectroscopy and Langmuir probe measurements are presented. The estimation procedure relies on measurements of the ratios of the intensities of the atomic triplet $3 p^{4} S^{0} \rightarrow 3 s^{4} P(742.36,744.23$, and $746.83 \mathrm{~nm})$ to the intensity of the 4-2 band of the first positive system $\left(A^{3} \Sigma_{u}^{+} \rightarrow B^{3} \Pi_{g}\right)$ at $750.39 \mathrm{~nm}$ and the measured relative vibrational distribution of the $B^{3} \Pi_{g}$ state. The electron energy distribution function, obtained from the second derivative of the Langmuir probe characteristic, and published excitation cross sections are used to calculate the electron-impact excitation rate coefficients-which are then compared to the atomic line and molecular band intensities to calculate the dissociation degree. For two distinct operating regimes, capacitively and inductively coupled, dissociation fractions of 5\% and $13 \%$ are obtained in the expansion region of a $10 \mathrm{mTorr}, 500 \mathrm{~W}, 10.74 \mathrm{MHz}$ helicon generated nitrogen plasma. The dissociation degree results suggest that the helicon source can provide excited molecular species for plasma assisted molecular beam epitaxy of III-group nitrides. () 2007 American Institute of Physics. [DOI: $10.1063 / 1.2777998]$
\end{abstract}

\section{INTRODUCTION}

Nitrogen and nitrogen-based mixture discharges are primarily employed in two classes of applications: (a) modification of surface properties by ion beam assisted deposition, plasma immersion ion implantation (such as steel surface hardening by nitridation), and deposition of hard compound films (such as $\mathrm{BN}, \mathrm{CN}, \mathrm{WN}$, and TiN for improving wear and corrosion resistance); (b) growth by plasma assisted molecular beam epitaxy (PAMBE) of wide band gap III-V group semiconductors such as $\mathrm{GaN}$ and nitrogen doping of different alloys such as GaNAs, InSbN, InGaNAs, and Ga$\mathrm{NAsSb}$ to obtain different heterostructures for UV/blue/green diodes or laser diodes. Each of these applications requires different nitrogen active species to be generated by the nitrogen plasma source. For surface modification, it has been shown that the quality of the surface and the thickness of the modified layer improve with increasing density and energy of atomic nitrogen ions in the plasma, ${ }^{1-3}$ i.e., surface modification applications require a nitrogen plasma with a high degree of dissociation. Conversely, for optimal $\mathrm{GaN}$ growth by PAMBE, the nitrogen plasma source should provide excited molecular states. Although the formation reaction of GaN has an energy of only $1.9 \mathrm{eV}$ and therefore all active nitrogen species, atomic or molecular, with an energy above this threshold may lead to the growth of GaN films, preliminary experiments suggest ${ }^{4-6}$ that the best species for growing

\footnotetext{
${ }^{a)}$ Author to whom correspondence should be addressed. Electronic mail: costel.biloiu@vsea.com
}

such films is the first excited neutral molecular state $A^{3} \Sigma_{u}^{+}$, which has an energy of $6.3 \mathrm{eV}$ (for $\nu=0$ ) above the ground state. The conclusion that the $A^{3} \Sigma_{u}^{+}$state is the best choice for film growth is based on three factors: (i) this species has a long radiative lifetime $(\sim 2.4-3.3 \mathrm{~s}$ for $\nu=0-6)$ (Ref. 7) and therefore survives long enough to travel from the plasma source to the MBE chamber; (ii) being neutral it will not be affected by the plasma sheath created in the front of the substrate and therefore will not induce damage in the growing film due to an excess of kinetic energy, as would happen with $\mathrm{N}^{+}$and $\mathrm{N}_{2}^{+}$which are accelerated by the potential drop across the sheath; and (iii) as suggested by experimental studies, ${ }^{4-6}$ being a molecule, it brings to the growing surface two atoms. In the ensuing dissociative chemisorption reaction, one of the nitrogen atoms binds to the surface while the second carries away the excess heat of reaction as kinetic energy. Thus, the reaction exothermicity is not dissipated through the GaN film. High residual stress in the film is avoided and better film quality is obtained. In contrast, the growth reaction involving atomic nitrogen is strongly exothermic with no means of dissipation other than through phonon creation, sputtering, or desorption-all of which promote poor nitrogen incorporation and point defect formation. Additional evidence that $A^{3} \Sigma_{u}^{+}$is the key active nitrogen species in growing high-quality $\mathrm{GaN}$ is provided by the spectral observations of Hughes $e t$ al. ${ }^{8}$ They showed that the best quality films and the highest deposition rates were obtained in plasmas for which the emission spectrum exhibited an intense first positive $\left(1^{+}\right)$system, a weak second positive $\left(2^{+}\right)$ system, and completely lack of emission from the first nega- 
tive $\left(1^{-}\right)$system. These spectral observations are consistent with a high population of the $B{ }^{3} \Pi_{g}$ state, and therefore the $A^{3} \Sigma_{u}^{+}$state as well, and the absence of molecular ions.

In our previous report, ${ }^{9}$ referenced as Part I throughout this paper, we described a helicon plasma source CHEWIE (Compact HElicon Waves and Instability Experiment) that might provide abundant molecular nitrogen excited species for GaN film growth. In this work, we examine the composition of the created plasma, with a specific focus on the degree of nitrogen dissociation $D$. Because of the large excitation rate coefficients for the $A^{3} \Sigma_{u}^{+}, B{ }^{3} \Pi_{g} W^{3} \Delta_{u}$, and $C^{3} \Pi_{u} \mathrm{~N}_{2}$ triplet states and the inevitable decay of the last three levels to the $A^{3} \Sigma_{u}^{+}$metastable, the $A^{3} \Sigma_{u}^{+}$state is likely to be the dominant metastable in the plasma. However, a large population of singlet metastable state $a^{\prime}{ }^{1} \Sigma_{u}^{-}(\sim 0.5 \mathrm{~s}$ radiative lifetime $)^{10}$ may also be present. Therefore, a low dissociation degree is indicative of a large molecular species population and implicitly a large $A^{3} \Sigma_{u}^{+}$metastable population.

In nitrogen plasmas, the dissociation degree can be estimated by quadrupole mass spectrometry (QMS). ${ }^{11,12}$ Since QMS involves plasma extraction and subsequent ionization of the nitrogen species, the signal detected by the quadrupole mass spectrometer is proportional to the species density in the ionizer. The species density in the ionizer is proportional to the species density in the plasma and the proportionality changes with changes in the temperature of the plasma and in the ionizer. ${ }^{12}$ A more direct measurement of the dissociation degree can be obtained through optical emission spectroscopy (OES). Vaudo et al. ${ }^{13}$ proposed the estimation of $D$ in nitrogen plasmas based on ratios of atomic lines to molecular bands intensities. Based on OES and QMS measurements, McCullogh et al. ${ }^{14,15}$ found a linear relationship between the ratio of the intensity of the $746.8 \mathrm{~nm}$ atomic line to the intensity of 4-2 band $(750.39 \mathrm{~nm})$ of the $1^{+}$system and the quantity $D /(1-D)$. Dissociation degrees as high as $67 \%$ for a 2 mTorr and $180 \mathrm{~W}$ input power microwave discharge were reported. A similar dependence of the ratio of the intensity of $744.2 \mathrm{~nm}$ atomic line to the intensity of the 4-2 band of the $2^{+}$system $(295.3 \mathrm{~nm})$ on the atomic/molecular population ratio was also reported by Sasaki et al. ${ }^{16}$ For a helicon generated plasma, they found that their empirical scaling relationship worked for electron densities up to $10^{13} \mathrm{~cm}^{-3}$. A monotonic dependence of the ratio of the intensities of an atomic line $(746.8 \mathrm{~nm})$ to the $0-0$ band $(337.1 \mathrm{~nm})$ of the second positive system on electron temperature in the range of $2-7 \mathrm{eV}$ was also reported by Itagaki et al. ${ }^{17}$ for a $915 \mathrm{MHz}$ electron cyclotron plasma. The same band of the $2^{+}$system in conjunction with the $174.4 \mathrm{~nm}$ atomic line intensity and rate coefficients calculated assuming a Maxwellian electron energy distribution function (eedf) was used by Nakano et al. ${ }^{18}$ to estimate the atomic/molecular population ratio. They found a ratio of $\sim 0.04$ for a 13.56 MHz, 5 mTorr, $1 \mathrm{~kW}$ input power inductively coupled plasma (ICP) discharge. With a model that accounted for the band intensities of the $\Delta \nu=-2$ sequence of the second positive system, the relative vibrational population of the $\nu$ $=0-3$ levels, and the intensity of the $746.8 \mathrm{~nm}$ line, Carrere et $a l .{ }^{19}$ reported calculated dissociation degrees as high as
$70 \%$ for a 0.01 SCCM (SCCM denotes cubic centimeter per minute at STP), $400 \mathrm{~W}$, nitrogen ICP discharge. They reported that by increasing the $\mathrm{N}_{2}$ flow rate, or by decreasing the input power, the dissociation fraction decreased. The maximum incorporation of nitrogen into GaInAsN was obtained for 0.2 SCCM and $200 \mathrm{~W}$ input power. Nitrogen dissociation in an ICP discharge was also investigated by Czerwiec et $a .^{12}$ by three methods: modulated beam mass spectrometry, the ratio of the atomic line intensity $(746.8 \mathrm{~nm})$ to $0-0$ band of the $2^{+}$system $(337.1 \mathrm{~nm})$ intensity, and actinometry using the Ar I $750.4 \mathrm{~nm}$ line. A dissociation degree of $\sim 60 \%-70 \%$ was obtained for a 50 mTorr, $5 \% \mathrm{Ar} / \mathrm{N}_{2}$ and $160-280 \mathrm{~W}$ input power discharge. Good agreement between the values obtained from mass spectrometry and actinometry was obtained, but the authors noted that the atomic to molecular line intensity ratio method gave nonrealistic values for the dissociation degree.

These previous experiments suggest that with an adequate model, the dissociation degree $(D)$ in nitrogen plasma can be accurately determined from OES observations. In this work, we present a method of D estimation based on the ratios of the intensities of the strong $\mathrm{N}$ I triplet $3 p{ }^{4} S^{0}$ $\rightarrow 3{ }^{4} P(742.36,744.23$, and $746.83 \mathrm{~nm})$ to the intensity of the 4-2 band of the first positive system $\left(A^{3} \Sigma_{u}^{+} \rightarrow B^{3} \Pi_{g}\right)$ at $750.39 \mathrm{~nm}$. We prefer to use the 4-2 band of $1^{+}$system instead of the commonly used $0-0$ or $4-2$ bands of the $2^{+}$system due to its convenient location near the triplet atomic lines; the 4-2 band overlaps the triplet lines and spans a narrow spectral range of $\sim 10 \mathrm{~nm}$. Therefore, corrections for the wavelength sensitivity of the optical chain are minimal. An even better option would be to use the atomic septet $3 p{ }^{4} P^{0} \rightarrow 3 s{ }^{4} P \quad(818.49,818.80,820.04,821.07,821.63$, 822.31 , and $824.24 \mathrm{~nm}$ ) transition sequence which has almost the same excitation energy $(11.84 \mathrm{eV})$ as the atomic triplet $(11.99 \mathrm{eV})$. Since that sequence includes seven different lines, it could provide a stronger constraint on the determination of $D$. However, the lack of strong $\mathrm{N}_{2}$ emission bands near this atomic septet (the 5-4 band of the $1^{+}$system at $820.48 \mathrm{~nm}$ is too weak for reliable measurements) prevents such analysis. To improve the accuracy of the calculated value of $D$, our 4-2 band-based method also accounts for the different excitation and deexcitation pathways and the relative vibrational distribution of the upper $B{ }^{3} \Pi_{g}$ electronic state.

\section{HELICON PLASMA SOURCE}

The experiments were performed in the CHEWIE helicon machine described in Part I. ${ }^{9}$ To maximize the population of molecular excited states in the diffusion chamber, the plasma source was designed with the inlet gas port at the junction of the helicon source-diffusion chamber (see Fig. 6 from Part 1). Pumping is accomplished at the bottom of the diffusion chamber. The expectation was that fresh gas molecules would act as a buffer for energetic electron and ion populations flowing downstream from the helicon plasma source, dissipating their energy in inelastic collisions and increasing the probability of three-body recombination. In Part I we showed that the gas temperature in the helicon 
source is roughly $100 \mathrm{~K}$ higher than in the diffusion chamber, $10 \mathrm{~cm}$ downstream from the junction. Although we do not have direct measurements of the electron density and temperature in the source, other researchers have reported electron temperatures of $5-7 \mathrm{eV}$ and electron densities of $(2-5) \times 10^{12} \mathrm{~cm}^{-3}$, corresponding to an ionization degree as high as $14 \%$, for similar operating parameters (pressure, magnetic field, and power) as our nitrogen helicon plasma source ${ }^{16,20} \mathrm{rf}$ discharges in pure nitrogen exhibit two distinct operation regimes: the dark regime, or capacitively coupled ( $E$ mode), for low input power and the bright regime, or inductively coupled ( $H$ mode), for relatively high input power. Typically, the transition from the $E$ mode to the $H$ mode is realized by increasing the input power. Occasionally, increasing the input power is sufficient to trigger a transition to the $H$ mode and the transition is facilitated by adding Ar, inserting a stabilizing electrode, ${ }^{12}$ or changing the magnetic field strength in the source. For an input power greater than $500 \mathrm{~W}$ in our source, increasing the magnetic field strength to about $800 \mathrm{G}$ triggers a transition to the $H$ mode. The source exhibits considerable hysteresis switching back to the $E$ mode at very small magnetic field strengths and then transitioning back to $H$ mode at magnetic field strengths as small as $20 \mathrm{G}$. For the measurements reported here, two operation regimes were investigated: the $E$ mode at $600 \mathrm{G}$ and $H$ mode at $850 \mathrm{G}$. All other parameters were held fixed: pressure of $10 \mathrm{mTorr}$, input power of $500 \mathrm{~W}$, and rf driving frequency of $10.74 \mathrm{MHz}$. Although our objective was to maximize the population of excited molecular states in the diffusion chamber (a plasma with a low dissociation degree), and since other groups have reported that $D$ scales inversely with nitrogen pressure, ${ }^{19}$ we worked at relatively low pressures. Note also that for PAMBE applications, the pressure in the Knudsen cell should not exceed $10^{-7}-10^{-8}$ mTorr. In fact, according to Ref. 8, the best quality GaN films and the highest deposition rates $(0.25-0.30 \mu \mathrm{m} / \mathrm{h})$ were obtained for very low operating pressure, $(2-5) \times 10^{-2}$ mTorr, downstream of an Oxford MPD21 rf plasma source.

\section{ELECTRON ENERGY DISTRIBUTION FUNCTION (EEDF) AND ELECTRON-IMPACT EXCITATION RATE COEFFICIENTS}

In estimating the nitrogen dissociation degree from a ratio of atomic line/molecular band intensities, a Maxwellian eedf is typically assumed. ${ }^{12,16,18}$ By choosing atomic lines and a molecular band that have approximately the same excitation threshold energy, e.g., atomic $3 p^{4} S^{0}$ level $(11.99 \mathrm{eV})$ and the $0-0(11.18 \mathrm{eV})$ or $4-2(12.12 \mathrm{eV})$ bands of the $2^{+}$system, the intensity ratios are easily calculated by assuming similar monotonic dependencies of the atomic and molecular electron-impact excitation rate coefficients on electron temperature. However, a sharply peaked excitation cross section (such as shown in Fig. 1) will result in a strong dependence of the electron-impact excitation rate coefficient on the electron temperature. Since the actual eedf in nitrogen plasmas typically has a bi-Maxwellian structure with two groups of electrons with two different temperatures ${ }^{21}$ an accurate estimation of the dissociation degree in a nitrogen plasma using OES techniques necessitates a complete mea-

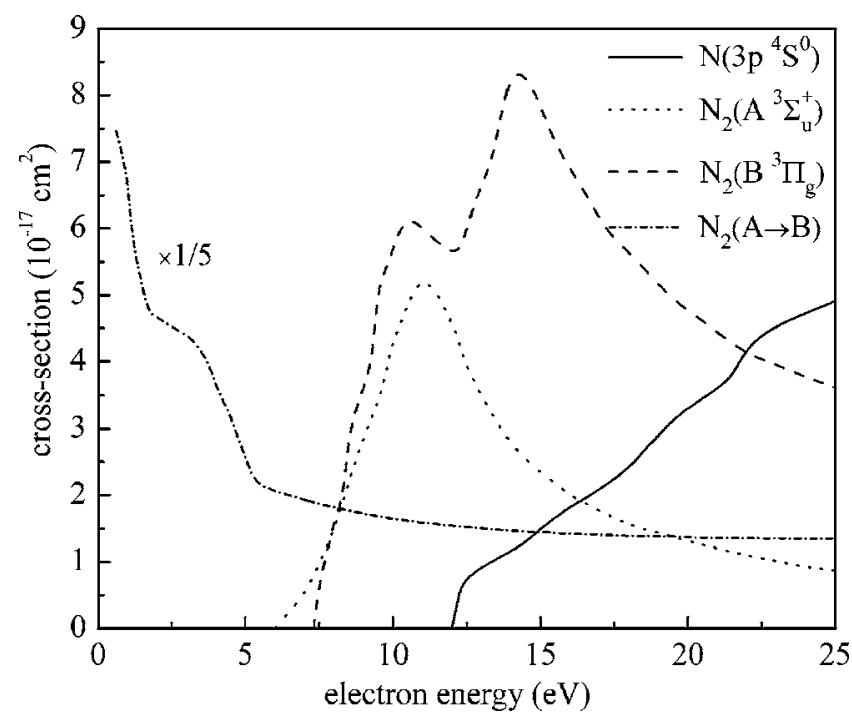

FIG. 1. Electron-impact excitation cross sections from ground level of the $\mathrm{N}\left(3 p^{4} S^{0}\right)$ atomic state (Ref. 27), $\mathrm{N}_{2}\left(A^{3} \Sigma_{u}^{+}\right)$(Ref. 33), and $\mathrm{N}_{2}\left(B^{3} \Pi_{g}\right)$ (Ref. 30) molecular electronic states, and $\mathrm{N}_{2}\left(B^{3} \Pi_{g}\right)$ from excited $\mathrm{N}_{2}\left(A^{3} \Sigma_{u}^{+}\right)$state (Ref. 34)

surement of the eedf. One advantage of a complete measurement of the eedf is the elimination of the requirement to use an atomic line-molecular band pair with nearly equal excitation energies. Therefore, in our case, by directly calculating the excitation rate coefficients based on available crosssection data and our measured eedf, the $\sim 3.7 \mathrm{eV}$ difference in excitation energies of the $\nu=4$ vibrational level of the $B$ state $(8.32 \mathrm{eV})$ and the $3 p^{4} S^{0}$ atomic level $(11.99 \mathrm{eV})$ does not adversely affect our determination of $D$.

\section{A. Langmuir probe analysis}

Electron energy distribution functions were obtained from Langmuir probe measurements through the Druyvestein probe characteristic differentiation method: ${ }^{22}$

$$
f(\varepsilon)=2\left(2 m_{e}\right)^{1 / 2}\left(e^{3} A\right)^{-1} d^{2} I_{p} / d V^{2},
$$

where $m_{e}$ and $e$ are the electron mass and charge, $A$ is the probe surface area, and $I_{p}$ and $V$ are the probe current and voltage. In Eq. (1), $f(\varepsilon)$ is the electron energy probability function (eepf) which is related to the electron energy distribution function, $F(\varepsilon)=\varepsilon^{1 / 2} f(\varepsilon)$. By integration, the electron density is

$$
n_{e}=\int_{0}^{\infty} F(\varepsilon) d \varepsilon
$$

and the effective electron temperature is

$$
T_{\text {eff }}=\frac{2}{3}\langle\varepsilon\rangle=\frac{2}{3 n_{e}} \int_{0}^{\infty} \varepsilon F(\varepsilon) d \varepsilon .
$$

Our rf compensated, cylindrical Langmuir probe consists of a $0.5 \mathrm{~mm}$ diameter graphite rod that extends $3 \mathrm{~mm}$ beyond an insulating alumina tube. The rf compensation is accomplished by a series of rf chokes covering the frequency range of 6-18 MHz and a $10 \mathrm{nF}$ shorting capacitor (see Ref. 23 for a detailed description of the probe). Probe measurements 
TABLE I. Rate coefficients for electron-impact excitation of $\mathrm{N}\left(3 p{ }^{4} S^{0}\right), \mathrm{N}_{2}\left(A{ }^{3} \Sigma_{u}^{+}\right)$, and $\mathrm{N}_{2}\left(B^{3} \Pi_{g}\right)$ states.

\begin{tabular}{cccccc}
\hline \hline$T_{e}(\mathrm{eV}) / k\left(\mathrm{~cm}^{3} \mathrm{~s}^{-1}\right)$ & $k_{\mathrm{N}}$ & $k_{\mathrm{N}_{2}}^{X \rightarrow A}$ & $k_{\mathrm{N}_{2}}^{A \rightarrow B}$ & $k_{\mathrm{N}_{2}}^{X \rightarrow B}$ & Obs. \\
\hline \multirow{2}{*}{3.3} & $1.84 \times 10^{-10}$ & $6.02 \times 10^{-10}$ & $1.78 \times 10^{-8}$ & $7.48 \times 10^{-10}$ & $\mathrm{a}$ \\
& $1.94 \times 10^{-10}$ & $1.33 \times 10^{-9}$ & $2.30 \times 10^{-8}$ & $1.85 \times 10^{-9}$ & $\mathrm{~b}$ \\
& $1.80 \times 10^{-10}$ & $5.73 \times 10^{-10}$ & $\cdots$ & $7.86 \times 10^{-10}$ & $\mathrm{c}$ \\
& & & & \\
4.1 & $7.75 \times 10^{-10}$ & $1.12 \times 10^{-9}$ & $1.80 \times 10^{-8}$ & $1.46 \times 10^{-9}$ & $\mathrm{a}$ \\
& $4.35 \times 10^{-10}$ & $2.20 \times 10^{-9}$ & $2.72 \times 10^{-8}$ & $3.73 \times 10^{-9}$ & $\mathrm{~b}$ \\
& $3.87 \times 10^{-10}$ & $9.80 \times 10^{-10}$ & $\cdots$ & $1.19 \times 10^{-9}$ & $\mathrm{c}$ \\
\hline
\end{tabular}

${ }^{\mathrm{a}}$ Calculated values based on experimentally obtained eedf's and the average cross section from independent particle model and Hartree-Fock-Slater calculations from Ref. 28 for $k_{\mathrm{N}}$; recommended theoretical cross sections from Ref. 31 for $k_{\mathrm{N}_{2}}^{X \rightarrow A}$ and $k_{\mathrm{N}_{2}}^{X \rightarrow B}$; theoretical cross section from Ref. 34 extrapolated up to $25 \mathrm{eV}$ for $k_{\mathrm{N}_{2}}^{A \rightarrow B}$. ${ }^{\mathrm{b}}$ Calculated values based on experimentally obtained eedf's and $R$-matrix cross section from Ref. 27 for $k_{\mathrm{N}}$; optical cross sections from Ref. 33 for $k_{\mathrm{N}_{2}}^{X \rightarrow A}$ and from Ref. 30 for $k_{\mathrm{N}_{2}}^{X \rightarrow B}$; theoretical cross section from Ref. 34 corrected for cascading effects for $k_{\mathrm{N}_{2}}^{A \rightarrow B}$.

${ }^{c}$ Interpolated values from Ref. 29 based on $R$-matrix calculated cross sections for $k_{\mathrm{N}}$; interpolated values from Ref. 32 obtained for a Maxwellian eedf and uncorrected for cascading effects for $k_{\mathrm{N}_{2}}^{X \rightarrow A}$ and $k_{\mathrm{N}_{2}}^{X \rightarrow B}$.

were taken on the axis of the diffusion chamber, $10 \mathrm{~cm}$ below the junction with the helicon source (see Fig. 6 in Part I). eepf's for the two different operational modes are shown in Fig. 2 instead eedf's since on a logarithmic plot a truly Maxwellian eepf is a straight line. ${ }^{24}$ In Fig. 2, both eepf's exhibit bi-Maxwellian distributions with the characteristic dip between 2 and $4 \mathrm{eV}$ due to the resonance in the nitrogen cross section associated with the formation of unstable $\mathrm{N}_{2}^{-25}$ In the $E$-mode case there is a cold electron population with a density of $n_{e c}=1.13 \times 10^{10} \mathrm{~cm}^{-3}$ and a hot electron population with a density of $n_{e h}=1.72 \times 10^{10} \mathrm{~cm}^{-3}$. The corresponding temperatures obtained from the slope, $T_{e}=-[d \ln f(\varepsilon) / d \varepsilon]^{-1}$, are $T_{e c}=2 \mathrm{eV}$ and $T_{e h}=4.5 \mathrm{eV}$. Integrating the entire distribution according to Eqs. (2) and (3) gives an effective temperature of $4.1 \mathrm{eV}$ and an overall density of 2.75 $\times 10^{10} \mathrm{~cm}^{-3}$. In the better confined $H$-mode plasmas, the increased number of electron-electron collisions and inelastic collisions with gas molecules results in a more nearly Maxwellian distribution. However, two electron populations are still observed: the cold population with a $2.4 \mathrm{eV}$ electron temperature and density of $1.52 \times 10^{10} \mathrm{~cm}^{-3}$ and the hot population with $3.7 \mathrm{eV}$ electron temperature and a density of $1.32 \times 10^{10} \mathrm{~cm}^{-3}$. Integrating the entire distribution yields a lower effective electron temperature of $3.3 \mathrm{eV}$ and slightly larger electron density, $2.86 \times 10^{10} \mathrm{~cm}^{-3}$.

\section{B. Electron-impact excitation rate coefficients}

The electron-impact excitation rate coefficients were computed based on available excitation cross-sections and the measured eedf according to

$$
k=\langle\sigma \nu\rangle=n_{e}^{-1} \sqrt{2 / m_{e}} \int_{0}^{\infty} \sigma(\varepsilon) \varepsilon^{1 / 2} F(\varepsilon) d \varepsilon
$$

As noted by Donnelly, ${ }^{26}$ when calculating rate coefficients it is better to use uncorrected optical cross sections instead theoretical ones, since the optically derived values include cascades from upper levels and excitation from lower metastable levels. As we show below, use of the optically derived cross sections greatly simplifies the rate equations. For electron-impact excitation of the $3 p^{4} S^{0}$ atomic level, no op- tical cross sections are available in the literature. However, recently Tayal and Zatsarinny ${ }^{27}$ published a set of cross sections for atomic nitrogen obtained by $R$-matrix calculations that include transitions between 39 atomic states. In Table I we present the rate coefficients for electron-impact excitation calculated using our experimentally determined eedf's and Ganas ${ }^{28}$ cross sections (obtained through generalized oscillator strength calculations), and the calculated Tayal and Zatsarinny cross sections. Also given in Table I are the rate coefficients obtained by Frost et al. ${ }^{29}$ through $R$-matrix calculations. For $3.3 \mathrm{eV}$ the rate coefficients are mutually consistent but for higher electron temperature $(4.1 \mathrm{eV})$ the rate coefficients calculated from the generalized oscillator strength calculations are roughly twofold larger than those using the $R$-matrix determined cross sections and the rate coefficient values of Frost $e t$ al. We note that Frost et al. ${ }^{29}$ included a few measured rate coefficients in their work which, after correction for electron-impact from metastable states, spontaneous decay from higher excited states, and electron-impact ionization, are in good agreement with $R$-matrix calculated values.

For calculation of electron-impact excitation rate coefficients for the $B{ }^{3} \Pi_{g}$ state, we used the optical cross sections of Stanton and St. John. ${ }^{30}$ Also shown in Table I are the rate coefficient values calculated using the recommended cross sections of Itikawa ${ }^{31}$ and calculated rate coefficients, uncorrected for cascading effects, published by Cartwright. ${ }^{32}$ All three values are similar but, as expected, the rate coefficients using the optical cross sections are somewhat larger than the other two values. Since our model includes the influence of the $A^{3} \Sigma_{u}^{+}$state on the population of the upper $B{ }^{3} \Pi_{g}$ state, the electron-impact rate coefficient for excitation from the molecule ground state was calculated using the measured cross sections of Borst. ${ }^{33}$ An important path for excitation to the $B^{3} \Pi_{g}$ state is electron excitation from the metastable $A^{3} \Sigma_{u}^{+}$state. Only $\mathrm{Huo}^{34}$ has reported calculated cross sections for this excited-to-excited state transition. In the $0-6 \mathrm{eV}$ energy range, this cross section is roughly four to seven times larger than peak values of the $B^{3} \Pi_{g}$ and $A^{3} \Sigma_{u}^{+}$ excitation cross sections from the ground state (see Fig. 2). Since the published values span only $0-12 \mathrm{eV}$, we fit the 

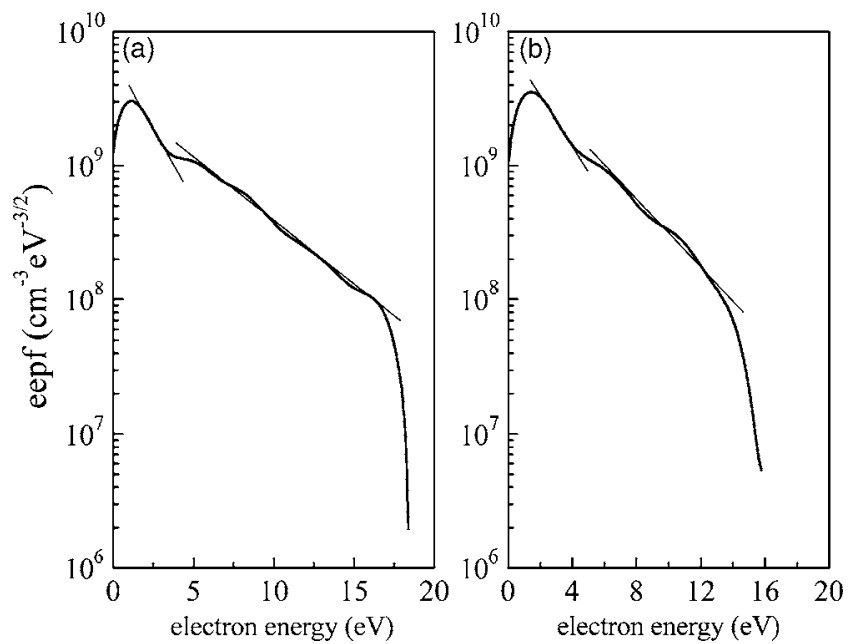

FIG. 2. Experimentally obtained electron energy probability functions for (a) $E$-mode and (b) $H$-mode operation regimes.

high energy tail of the published values with an exponential function and extrapolated values up to $25 \mathrm{eV}$. The extrapolation has a minimal effect on the calculated rate coefficient since the calculation is dominated by the cross-section values in the range of $0-6 \mathrm{eV}$, where the eedf and the cross section are both largest.

The optical cross-section values for excitation of $A^{3} \Sigma_{u}^{+}$ and $B^{3} \Pi_{g}$ states from ground state are roughly 2.5 times larger (peak values) than corresponding theoretical values, ${ }^{35}$ which is why the rate coefficients in Table I based on the optically derived values are larger than those obtained using the theoretical cross sections. Therefore, to adjust all the calculated rate coefficients to reflect the increased magnitudes that would be obtained if optically derived cross sections were available for all the transitions, and to account for the strong cascading effect due to emission from the $1^{+}$system, the rate coefficients calculated using the cross sections given in Ref. 34 were multiplied by factors of 2.3 and 2.7 for the 3.3 and $4.1 \mathrm{eV}$ cases, respectively (the average of the scaling factors obtained for $A^{3} \Sigma_{u}^{+}$and $B^{3} \Pi_{g}$ states). As we show below, this scaling correction barely affects the calculated degree of dissociation.

\section{ATOMIC LINES AND MOLECULAR BAND INTENSITIES IN EMISSION}

\section{A. Atomic line intensity}

The 742.36, 744.23, and $746.83 \mathrm{~nm}$ triplet lines are among the brightest lines of atomic nitrogen in low temperature plasmas. Since they are transitions from the same upper level $\left(3 p{ }^{4} S^{0}\right)$ to the same lower level $\left(3 s^{4} P\right)$ (see Table II), comparative analysis of their emission intensities is relatively simple, i.e., the emitted intensities are determined by the branching ratio. The emitted intensity of a transition from the upper level $j$ to the lower level $k$ may be written as

$$
I_{\mathrm{N}}^{j k}=\alpha\left(h c / \lambda_{j k}\right) S\left(\lambda_{j k}\right) b_{j k}\left[N_{j}\right],
$$

where $\alpha$ is a constant depending on the characteristics of the collection optics, $S$ the sensitivity of the optical chain [window, lens, optical fiber, spectrometer, and charge-coupled device (CCD) camera], $\left[N_{j}\right]$ the population of the upper emitting state, and $h, c$, and $\lambda$ have the usual meaning of Planck's constant, the speed of light in vacuum, and the radiation wavelength, respectively. The quantity $b_{j k}$ is the branching ratio of the transition $j \rightarrow k$ defined as ${ }^{36}$

$$
b_{j k}=\frac{A_{j k}}{\sum_{k^{\prime}} A_{j k^{\prime}}},
$$

where $A_{j k}$ is the transition probability and the summation is over all allowed transitions $j \rightarrow k^{\prime}$. In Eq. (5) we did not include the quantum yield for spontaneous emission (the radiative decay rate divided by the total decay rate $)^{26,37}$ since, as we will show later, the spontaneous emission and the other channels of deexcitation are already included in the overall excitation rate coefficient. The excited level population density $\left[N_{j}\right]$ is given by the rate balance equation

$$
d\left[N_{j}\right] / d t \cong k_{N} n_{e}\left[\mathrm{~N}\left({ }^{4} S\right)\right]-\left[N_{j}\right] / \tau_{N}^{\mathrm{rad}}-k_{N}^{q}\left[N_{j}\right]\left[\mathrm{N}_{2}(X)\right],
$$

where $k_{\mathrm{N}}$ is the electron-impact excitation rate coefficient for direct excitation from ground state, $n_{e}$ the electron density, $\left[\mathrm{N}\left({ }^{4} S\right)\right]$ the population of the ground atomic state $\left(2 p^{4} S^{0}\right)$, $\left[\mathrm{N}_{2}(X)\right]$ the population of the molecular ground level, and $\tau_{\mathrm{N}}^{\mathrm{rad}}=26.3 \mathrm{~ns}$ the radiative lifetime ${ }^{38}$ of the $\mathrm{N}\left(3 p^{4} S^{0}\right)$. Note that in Eq. (7) we neglected other channels for population such as excitation from the metastable states of $\mathrm{N}\left(3 p^{2} P^{0}\right)$ and $\mathrm{N}\left(3 p^{2} D^{0}\right)(2.38$ and $3.58 \mathrm{eV}$, respectively, above the ground state), ${ }^{39}$ dissociative excitation, cascading from upper levels, or loses due to ionization through electron collisions. Assuming a Maxwellian eedf, Czerwiec et al. ${ }^{12}$ calculated the rate coefficients for the first three processes and found that excitation from metastable states and dissociative excitation is one to two orders of magnitude smaller than excitation rates from the ground state for electron temperatures larger than $2 \mathrm{eV}$. Given that the metastable state population is six to ten times smaller than the ground state population, ${ }^{29}$ the production rate due to these processes is between two and three orders of magnitude smaller than the production rate by electron impact of the ground state. Similar conclusions were

\begin{tabular}{|c|c|c|c|c|c|c|}
\hline \multirow[b]{2}{*}{$\lambda(\mathrm{nm})$} & \multicolumn{2}{|c|}{ Upper level $j$} & \multicolumn{2}{|c|}{ Lower level $k$} & \multirow{2}{*}{$\begin{array}{c}A_{j k} \\
\left(\times 10^{6} \mathrm{~s}^{-1}\right)\end{array}$} & \multirow[b]{2}{*}{$b_{j k}$} \\
\hline & $E\left(\mathrm{~cm}^{-1}\right)$ & $J$ & $E\left(\mathrm{~cm}^{-1}\right)$ & $J$ & & \\
\hline 742.36 & 96750.84 & $3 / 2$ & 83284.07 & $1 / 2$ & 5.95 & 0.158 \\
\hline 744.23 & 96750.84 & $3 / 2$ & 83317.83 & $3 / 2$ & 12.4 & 0.329 \\
\hline 746.83 & 96750.84 & $3 / 2$ & 83364.62 & $5 / 2$ & 19.3 & 0.513 \\
\hline
\end{tabular}
reported by Levanton et al. ${ }^{40}$ for the $3 s^{4} P$ and $2 p^{4} P$ levels

TABLE II. Spectral characteristics of the N I $3 p^{4} S^{0} \rightarrow 3 s^{4} P$ triplet lines (Ref. 39). 


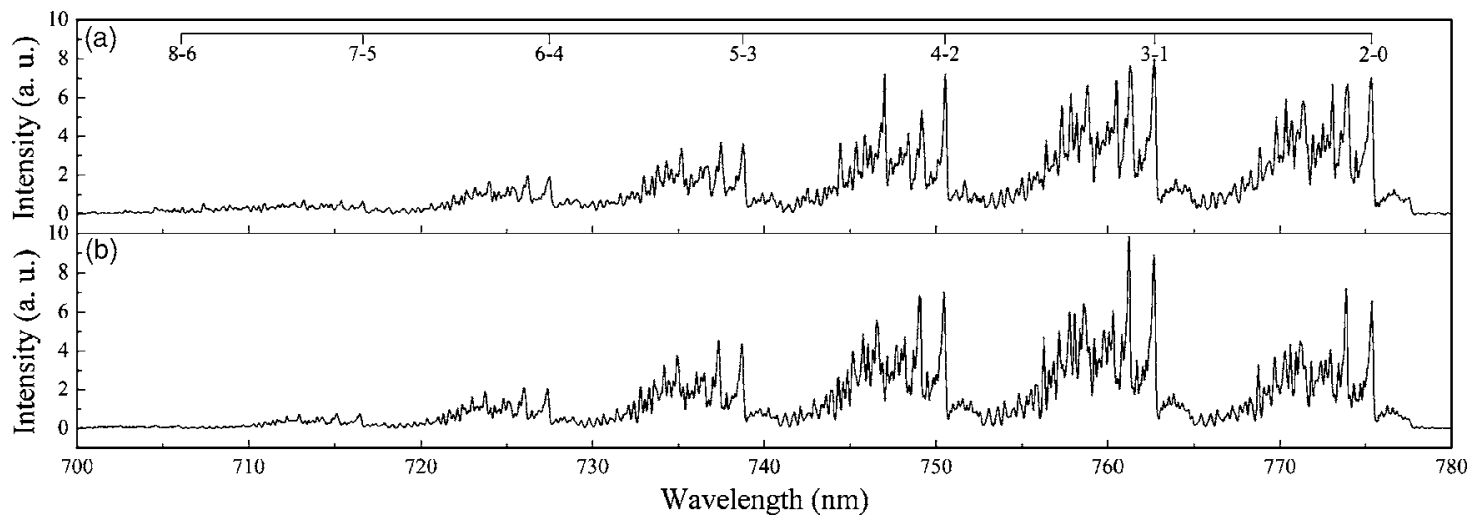

FIG. 3. (a) Experimentally obtained $\Delta v=2$ band sequence of the first positive system; (b) synthetically generated sequence for the best fit vibrational temperature.

which lie at 10.33 and $10.94 \mathrm{eV}$, respectively, i.e., 1.7 and $1 \mathrm{eV}$ below the $3 p^{4} S^{0}$ level.

Although at the low pressure used in our experiments, collisional quenching of the $3 p^{4} S^{0}$ atomic state should be minimal, Catherinot and $\mathrm{Sy}^{41}$ reported efficient quenching of the $3 p^{4} S^{0}$ state by $\mathrm{N}_{2}(X, \nu \geqslant 2)\left(k_{\mathrm{N}}^{q}=6.5 \times 10^{-9} \mathrm{~cm}^{3} \mathrm{~s}^{-1}\right)$. The quenching resulted in a ground state atom and an excited molecule in the singlet state $\mathrm{N}_{2}\left(b{ }^{1} \Pi_{u}\right)$ or higher singlet states $\mathrm{N}_{2}\left(b^{\prime}{ }^{1} \Sigma_{u}^{+}, c_{n}{ }^{1} \Pi_{u}\right)$. For this measured rate coefficient and assuming $50 \%$ of the molecules in the ground state are in $\nu \geqslant 2$ vibrational levels at a pressure of $10 \mathrm{mTorr}$, the resultant quenching frequency of $\sim 1.3 \times 10^{6} \mathrm{~s}^{-1}$ is $\sim 5 \%$ of the radiative loss frequency, $3.8 \times 10^{7} \mathrm{~s}^{-1}$. Since collisional quenching increases with increasing gas pressure, these calculations indicate that collisional quenching is significant only for gas pressures above 20 mTorr. In steady state, Eq. (7) becomes

$$
\left[N_{j}\right]=K_{N} n_{e}\left[\mathrm{~N}\left({ }^{4} S\right)\right] \tau_{\mathrm{N}}^{\mathrm{rad}}
$$

where $K_{\mathrm{N}}=k_{\mathrm{N}} /\left\{1+k_{\mathrm{N}}^{q}\left[\mathrm{~N}_{2}(X)\right] \tau_{\mathrm{N}}^{\mathrm{rad}}\right\}$ is the total excitation rate coefficient, including the correction for quenching collisions.

\section{B. Molecular band intensity}

The emission intensity for the $\nu^{\prime}=4 \rightarrow \nu^{\prime \prime}=2$ molecular band of the $1^{+}$system $\left(B^{3} \Pi_{g} \rightarrow A^{3} \Sigma_{u}^{+}\right)$is

$$
I_{\mathrm{N}_{2}}^{\nu^{\prime} \nu^{\prime \prime}}=\alpha\left(h c / \lambda_{\nu^{\prime} \nu^{\prime \prime}}\right) S\left(\lambda_{\nu^{\prime} \nu^{\prime \prime}}\right) q_{\nu^{\prime}} b_{\nu^{\prime} \nu^{\prime \prime}}\left[\mathrm{N}_{2}^{\nu^{\prime}=4}\right]
$$

where $\lambda_{\nu^{\prime} \nu^{\prime \prime}}=748.4 \mathrm{~nm}$ is the 4-2 band origin wavelength and $\left[\mathrm{N}_{2}^{\nu \prime}=4\right]$ is the population of the $\nu^{\prime}=4$ vibrational level of the $B^{3} \Pi_{g}$ state. As was done with the atomic line intensity analysis, the quantum yield of the emitting vibrational level was omitted. Since there are no selection rules for vibrational transitions, the summation in denominator of the branching ratio $b_{\nu^{\prime} \nu^{\prime \prime}}$ of the $4 \rightarrow 2$ vibrational transition includes all energetically possible lower vibrational levels ${ }^{42}$

$$
b_{\nu^{\prime} \nu^{\prime \prime}}=b_{42}=\frac{A_{4 \rightarrow 2}}{\sum_{\nu^{\prime \prime}=0}^{12} A_{4 \rightarrow \nu^{\prime \prime}}},
$$

where $A_{4 \rightarrow \nu^{\prime \prime}}=\left(64 \pi^{4} / 3 h \lambda^{3}\right) q_{4 \rightarrow \nu^{\prime \prime}}\left|R_{e}\left(r_{4 \rightarrow \nu^{\prime \prime}}\right)\right|^{2}$ is the band transition probability [with $q_{4 \rightarrow \nu^{\prime \prime}}$ and $\left|R_{e}\left(r_{4 \rightarrow \nu^{\prime \prime}}\right)\right|^{2}$ the
Franck-Condon factor and electronic-vibrational transition moment, respectively]. ${ }^{43,44}$ In Eq. (10), $q_{\nu^{\prime}}$ is the weighted Frank-Condon factor ${ }^{33,45}$ for excitation from the molecule ground state to $\nu^{\prime}=4$ of the $B{ }^{3} \Pi_{g}$ electronic state. The weighted Frank-Condon factor arises from the relationship between the emission cross section and the electron-impact excitation cross section: ${ }^{31}$

$$
\sigma_{\nu^{\prime} \nu^{\prime \prime}}^{e m}=b_{\nu^{\prime} \nu^{\prime \prime}} \sigma_{\nu^{\prime}}^{e m}=b_{\nu^{\prime} \nu^{\prime \prime}} q_{\nu^{\prime}} \sigma^{\mathrm{exc}}
$$

Since electron excitation may occur from any vibrational level of the $X^{1} \Sigma_{g}^{+}$state, the vibrational distribution of the $X$ state is needed to determine the appropriate weighting. As proposed by Levanton et al. ${ }^{46}$ as a zero order approximation, we used our experimentally measured relative vibrational distribution of the $B^{3} \Pi_{g}$ state and the available $X \rightarrow B$ FrankCondon factors ${ }^{10}$ to determine the relative vibrational distribution of the $X$ state.

The population in the $\nu^{\prime}=4$ vibrational level can be expressed as a fraction of the entire population of the $B{ }^{3} \Pi_{g}$ electronic state by using the experimentally determined vibrational distributions of the $B{ }^{3} \Pi_{g}$ state $\left[\mathrm{N}_{2}\left(B, \nu^{\prime}=4\right)\right]$ $=f_{\nu^{\prime}=4}\left[\mathrm{~N}_{2}(B)\right]$. As we have shown previously, ${ }^{47}$ rotational temperature, vibrational temperature, and the vibrational distribution of $\mathrm{N}_{2}$ can be obtained by comparison of OES measurements with a complete model of the state populations (see Fig. 3). However, the vibrational distribution can be obtained directly and quickly by integrating the band intensities and accounting for spectral sensitivity and band origin wavelength. As shown in Fig. 4(a), vibrational distributions were obtained from the sequences $\Delta \nu=0-\Delta \nu=+5$. After neglecting the bands with poor signal-to-noise ratios (the last one or two weak bands in each sequence, e.g., 7-5 and 8-6 in Fig. 3) in the Boltzmann plot of the averaged values corresponding to each $\nu$, a vibrational temperature of $(6140 \pm 210) \mathrm{K}$ is obtained for the $H$ mode and slightly lower temperature $(5680 \pm 180) \mathrm{K}$ for the $E$ mode. These vibrational temperature values are consistent with other reported values for low pressure nitrogen plasmas. ${ }^{48,49}$ Qualitatively, the calculated relative vibrational distributions of the ground state [as shown in Fig. 4(b)] resemble those reported for pulsed dc nitrogen discharges (Figs. 6 and 7 in Ref. 46). The 

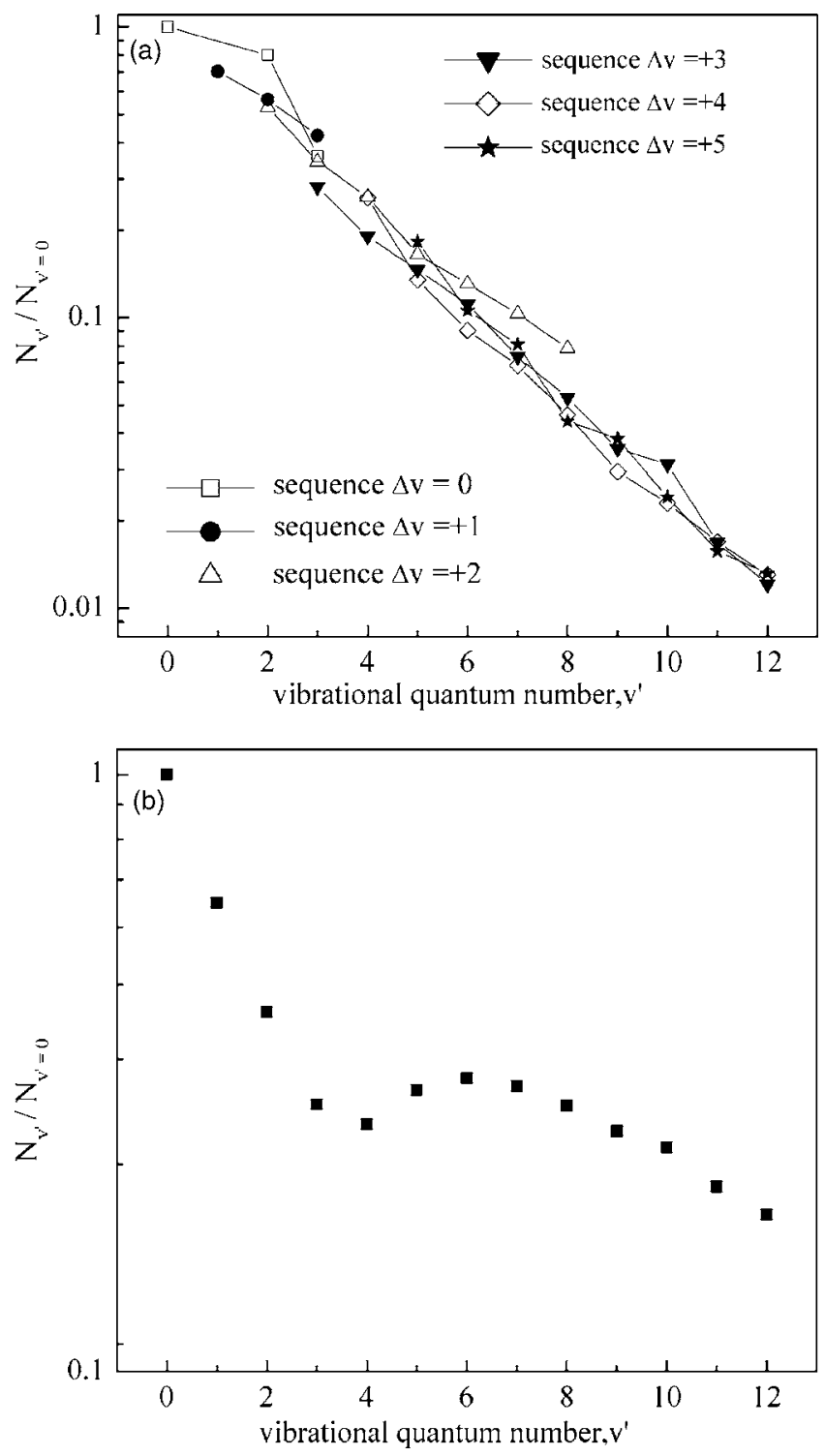

FIG. 4. Relative vibrational distributions of (a) $\mathrm{N}_{2}\left(B{ }^{1} \Pi_{g}\right)$ electronic state obtained from integrated band intensities of $\Delta v=0-\Delta v=5$ sequences; (b) $\mathrm{N}_{2}\left(X^{1} \Sigma_{g}^{+}\right)$electronic state calculated based on relative vibrational distribution of $\mathrm{N}_{2}\left(B^{3} \Pi_{g}\right)$ electronic state and $B$ - $X$ Franck-Condon factors.

most significant difference is in the position of the characteristic minimum, most probably an effect of the much lower pressure used in these experiments.

The next step is to relate the excited state population $\left[\mathrm{N}_{2}(B)\right]$ to the molecule ground state population $\left[\mathrm{N}_{2}(X)\right]$. This can be done by taking into consideration the different channels that compete in the population of the $\mathrm{N}_{2}\left(B{ }^{3} \Pi_{g}\right)$ state. The population of the upper $B{ }^{3} \Pi_{g}$ state is a result of a balance between many excitation and deexcitation processes. The main processes are shown below:

$$
\begin{aligned}
& e+\mathrm{N}_{2}(X) \rightarrow e+\mathrm{N}_{2}(B), \\
& e+\mathrm{N}_{2}(A) \rightarrow e+\mathrm{N}_{2}(B), \\
& \mathrm{N}_{2}(A)+\mathrm{N}_{2}(X, 5 \leqslant \nu \leqslant 14) \rightarrow \mathrm{N}_{2}(X)+\mathrm{N}_{2}(B), \\
& \mathrm{N}_{2}(A)+\mathrm{N}_{2}(A) \rightarrow \mathrm{N}_{2}(X)+\mathrm{N}_{2}(B),
\end{aligned}
$$

$$
\begin{aligned}
& \mathrm{N}_{2}(C) \rightarrow \mathrm{N}_{2}(B)+h \nu\left(2^{+} \text {system }\right), \\
& \mathrm{N}\left({ }^{4} S\right)+\mathrm{N}\left({ }^{4} S\right)+\mathrm{N}_{2}(X) \rightarrow \mathrm{N}_{2}(X)+\mathrm{N}_{2}(B), \\
& \mathrm{N}_{2}(X, \nu \geqslant 14)+\mathrm{N}_{2}(X, \nu \geqslant 14) \rightarrow \mathrm{N}_{2}(X)+\mathrm{N}_{2}(B), \\
& \mathrm{N}_{2}(B) \rightarrow \mathrm{N}_{2}(A)+h \nu\left(1^{+} \text {system }\right), \\
& \mathrm{N}_{2}(B)+\mathrm{N}_{2}(X) \rightarrow \mathrm{N}_{2}(A)+\mathrm{N}_{2}(X), \\
& \mathrm{N}_{2}(B)+\mathrm{N}_{2}(X) \rightarrow \mathrm{N}_{2}(X)+\mathrm{N}_{2}(X) .
\end{aligned}
$$

Under steady-state conditions, the population channels [processes (12)-(18)] will balance the depopulation channels [processes (19)-(21)] and the population of the $B{ }^{3} \Pi_{g}$ state is obtained from the rate balance equation. However, many of the processes listed above lead to small rates of population change and can be neglected. In calculating the electronimpact excitation rates [processes (12) and (13)] we used the worst-case scenario, i.e., the smallest electron-impact rate coefficients corresponding to $T_{e}=3.3 \mathrm{eV}$ (see Table I). The estimated rates for the processes (12) and (13) are 1.36 $\times 10^{16}$ and $1.54 \times 10^{13} \mathrm{~cm}^{-3} \mathrm{~s}^{-1}$, respectively. In estimating the process (13) and other rates involving excited triplet states, we considered a relative population of the excited levels in the ratio $\left[\mathrm{N}_{2}(X)\right]:\left[\mathrm{N}_{2}(A)\right]:\left[\mathrm{N}_{2}(B)\right]:\left[\mathrm{N}_{2}(C)\right]=1: 9$ $\times 10^{-5}: 8 \times 10^{-7}: 2 \times 10^{-10}$ based on experimentally determined values for the $\nu=0-2$ levels given in Ref. 50. The reported rate coefficients for associative excitation (14) and the pooling reaction (15) are $2 \times 10^{-11}$ and 7.7 $\times 10^{-11} \mathrm{~cm}^{3} \mathrm{~s}^{-1}$, respectively. ${ }^{51}$ Using the same relative population of the excited levels used to estimate the (13) rate, the pooling process leads to a negligible population rate of $4.58 \times 10^{10} \mathrm{~cm}^{-3} \mathrm{~s}^{-1}$. For a reasonable value of $5 \%$ of $\mathrm{N}_{2}(X)$ molecules in $\nu \geqslant 5$, associative excitations yield a population rate of $6.5 \times 10^{12} \mathrm{~cm}^{-3} \mathrm{~s}^{-1}$. Reaction (16) may be excluded since we are using optically derived cross sections. The three-body recombination process (17) with a rate coefficient $^{51}$ of $8.27 \times 10^{-34} \exp \left(500 / T_{\text {gas }}\right) \mathrm{cm}^{6} \mathrm{~s}^{-1}$ yields a total rate of only $6.4 \times 10^{8} \mathrm{~cm}^{-3} \mathrm{~s}^{-1}$ for an upper bound dissociation degree of $50 \%$. The spin forbidden reaction (18) that involves $\mathrm{N}_{2}(X, \nu \geqslant 14)$ can also be neglected because of its low rate coefficient $\left(10^{-15} \mathrm{~cm}^{3} \mathrm{~s}^{-1}\right)$ (Ref. 52) and the small population of high vibrational levels. The only process that significantly contributes to the destruction of the $B$ state is radiative deexcitation (radiative lifetime $\sim 4-12 \mu \mathrm{s}$ ) (Ref. 53) since the quenching by collisions with molecules in the ground state $\left[(20)\right.$ and (21)] leading to formation of $\mathrm{N}_{2}(A)$ $\left(k=2.85 \times 10^{-11} \mathrm{~cm}^{3} \mathrm{~s}^{-1}\right)$ (Ref. 51) or high vibrational level $\mathrm{N}_{2}(X)\left(k=1.5 \times 10^{-12} \mathrm{~cm}^{3} \mathrm{~s}^{-1}\right)$ (Ref. 51) yields total rates of $1.7 \times 10^{12}$ and $1.1 \times 10^{11} \mathrm{~cm}^{-3} \mathrm{~s}^{-1}$, respectively, more than one order of magnitude smaller than radiative deexcitation. Predissociation, which is another channel for deexcitation, but without radiative emission, can also be neglected since predissociation of $\mathrm{N}_{2}\left(B^{3} \Pi_{g}\right)$ only manifests for vibration levels above $\nu=12 .^{10,54}$ Our estimates of the various population rates are consistent with the predictions of a plasma kinetic model ${ }^{55}$ for a 100 mTorr discharge in pure nitrogen with a gas temperature of $350 \mathrm{~K}$, an electron temperature of 
$\sim 1-2 \mathrm{eV}$, and an electron density of $4 \times 10^{10} \mathrm{~cm}^{-3}$. The model predicts that the main population channels for the $B{ }^{3} \Pi_{g}$ state are electron impact from the molecule ground state, which accounts for $\sim 55 \%$ of the total production rate, and electron-impact excitation from the metastable $A^{3} \Sigma_{u}^{+}$ state, which accounts for $\sim 34 \%$. According to the model, the other population channels have a modest contribution: radiative decay of the upper $C^{3} \Pi_{u}$ state $\sim 7 \%$, associative excitation $\sim 3 \%$, and the pooling reaction $\sim 1 \%$. Disregarding all production processes having rates below $1 \times 10^{13} \mathrm{~cm}^{-3} \mathrm{~s}^{-1}$, the rate equation for the $B$ state is

$$
\begin{aligned}
d\left[\mathrm{~N}_{2}(B)\right] / d t \cong & k_{\mathrm{N}_{2}}^{X \rightarrow B} n_{e}\left[\mathrm{~N}_{2}(X)\right]+k_{\mathrm{N}_{2}}^{A \rightarrow B} n_{e}\left[\mathrm{~N}_{2}(A)\right] \\
& -\left[\mathrm{N}_{2}(B)\right] / \tau_{\mathrm{N}_{2}}^{\mathrm{rad}},
\end{aligned}
$$

where $k_{\mathrm{N}_{2}}^{X, A \rightarrow B}$ are the electron-impact excitation rate coefficients for excitation of the $B^{3} \Pi_{g}$ state from $X^{1} \Sigma_{g}^{+}$and $A^{3} \Sigma_{u}^{+}$ states, respectively, and $\tau_{\mathrm{N}_{2}}^{\text {rad }}$ is the weighted radiative lifetime of the $B{ }^{3} \Pi_{g}$ state obtained from the $\nu=0-12$ level lifetimes ${ }^{53}$ and the experimentally measured relative vibrational population distribution.

For the electronic state $A^{3} \Sigma_{u}^{+}$, in addition to the processes (13)-(15), (19), and (20) already described, other significant creation and destruction processes are

$$
\begin{aligned}
& e+\mathrm{N}_{2}(X) \rightarrow e+\mathrm{N}_{2}(A), \\
& \mathrm{N}\left({ }^{4} S\right)+\mathrm{N}\left({ }^{4} S\right)+\mathrm{N}_{2}(X) \rightarrow \mathrm{N}_{2}(X)+\mathrm{N}_{2}(A), \\
& \mathrm{N}_{2}(X, \nu \geqslant 12)+\mathrm{N}_{2}(X, \nu \geqslant 12) \rightarrow \mathrm{N}_{2}(X)+\mathrm{N}_{2}(A), \\
& \mathrm{N}_{2}(A)+\mathrm{N}\left({ }^{4} S\right) \rightarrow \mathrm{N}_{2}(X)+\mathrm{N}\left({ }^{2} P\right), \\
& \mathrm{N}_{2}(A) \stackrel{\text { diff }}{\longrightarrow} \mathrm{N}_{2}(X), \\
& \mathrm{N}_{2}(A)+\mathrm{N}_{2}(X) \rightarrow \mathrm{N}_{2}(X)+\mathrm{N}_{2}(X), \\
& \mathrm{N}_{2}(A)+\mathrm{N}_{2}(A) \rightarrow \mathrm{N}_{2}(X)+\mathrm{N}_{2}(C), \\
& \mathrm{N}_{2}(A) \rightarrow \mathrm{N}_{2}(X)+h \nu \quad \text { (Vergand-Kaplan system). }
\end{aligned}
$$

The principal population channel is electron-impact excitation from the ground state (23) with a total estimated rate of $9.8 \times 10^{15} \mathrm{~cm}^{-3} \mathrm{~s}^{-1}$. As we have shown previously, the formation of $\mathrm{N}_{2}(A)$ through (14), (15), and (20) processes is negligible compared to the (23) process. Process (19), although efficient in the creation of the $A$ state, ${ }^{45,56}$ can be neglected because we are using "corrected" cross sections. The three-body recombination process (24) with a rate coefficient ${ }^{56}$ of $2.4 \times 10^{-33} \mathrm{~cm}^{6} \mathrm{~s}^{-1}$ yields a production rate of $3.85 \times 10^{10} \mathrm{~cm}^{-3} \mathrm{~s}^{-1}$ (assuming a $50 \%$ dissociation degree) and can therefore be neglected. For the same reasons as noted for the $B^{3} \Pi_{g}$ state, the spin forbidden reaction (25) $\left(k=1.5 \times 10^{-13} \mathrm{~cm}^{3^{g}} \mathrm{~s}^{-1}\right)$ (Ref. 52) makes a negligible contribution. For depopulation of the $A^{3} \Sigma_{u}^{+}$state, there are three processes with approximately equal contributions: quenching by collisions with atoms in the ground state (26), diffusive losses (27), and collisions with electrons. For a rate coefficient ${ }^{57}$ of $k_{\mathrm{N}_{2}}^{q}=4 \times 10^{-11} \mathrm{~cm}^{3} \mathrm{~s}^{-1}$ and a dissociation de- gree of $50 \%$, the loss rate due to quenching by collisions with atoms in the ground state is $2.61 \times 10^{14} \mathrm{~cm}^{-3} \mathrm{~s}^{-1}$. Losses due to the diffusion to the walls were estimated from the characteristic diffusion time, ${ }^{58}$

$$
\frac{1}{\tau_{\mathrm{N}_{2}}^{D}}=\frac{D_{0}}{\left[\mathrm{~N}_{2}\right] \Lambda^{2}}\left(\frac{T_{\mathrm{gas}}}{300}\right)^{3 / 2},
$$

where $D_{0}=5 \times 10^{18} \mathrm{~cm}^{-1} \mathrm{~s}^{-1}$ is the reduced diffusion coefficient, ${ }^{51}\left[\mathrm{~N}_{2}\right]$ is the total nitrogen density at $10 \mathrm{mTorr}$; $\Lambda$ the characteristic diffusion length $\left(1 / \Lambda^{2}=(2.405 / R)^{2}\right.$ $+(\pi / L)^{2}$, with $R$ and $L$ the chamber radius and length, respectively, 2.405 the first root of the zeroth order Bessel function; and $T_{\text {gas }}$ is the gas temperature. For our diffusion chamber $(R=7.5 \mathrm{~cm}, L=30 \mathrm{~cm}), \Lambda \cong 4.65 \mathrm{~cm}$ and the estimated diffusion loss rate is $2.11 \times 10^{14} \mathrm{~cm}^{-3} \mathrm{~s}^{-1}$.

The third process leading to the destruction of the $\mathrm{N}_{2}(A)$ state is collisions with electrons and, as discussed previously in regard to the $\mathrm{N}_{2}(B)$ state [process (13)], the depopulation loss rate due to electron collisions is $1.54 \times 10^{13} \mathrm{~cm}^{-3} \mathrm{~s}^{-1}$. Electron collisional deactivation of the $\mathrm{N}_{2}(A)$ state results not only in the production of the $\mathrm{N}_{2}(B)$ state but other states as well, e.g., $W^{3} \Delta_{u}, B^{\prime}{ }^{3} \Sigma_{u}^{-}, C^{3} \Pi_{u}$, and $E^{3} \Sigma_{g}^{+}$. Cross sections for these processes are not available in the literature. However, since all these states lie between 0.8 and $4.5 \mathrm{eV}$ above the $B{ }^{3} \Pi_{g}$ state, except $W^{3} \Delta_{u}$ (which is only $0.01 \mathrm{eV}$ above and actually cannot be separated from $B^{3} \Pi_{g}$ state due to intrasystem cascading - the Wu-Benesch infrared system), since the $B{ }^{3} \Pi_{g}$ state is $1.2 \mathrm{eV}$ further above the $A^{3} \Sigma_{u}^{+}$state, and since the eedf is peaked at low energy, it is reasonable to assume that the (13) process is the main channel for electron collision deactivation. Another possible loss process is quenching by collisions with molecules (28). Here the spread of data in reported rate coefficient values spans six orders of magnitude. Values of $2.6 \times 10^{-18}$ and $3.8 \times 10^{-17} \mathrm{~cm}^{3} \mathrm{~s}^{-1}$ for $\nu=0$ and $\nu=1$, respectively, were reported by Levron and Phelps. ${ }^{59}$ Frequently used in nitrogen plasma modeling is a value of $3 \times 10^{-16} \mathrm{~cm}^{3} \mathrm{~s}^{-1}$ (based on Ref. 60-which, in fact, does not report measurements of this particular rate coefficient but itself cites the Levron and Phelps paper). At the higher end, a value of $3.7 \times 10^{-12} \mathrm{~cm}^{3} \mathrm{~s}^{-1}$ was reported by Piper. $^{57}$ Assuming a midrange value of $k=3.1$ $\times 10^{-15} \mathrm{~cm}^{3} \mathrm{~s}^{-1}$ results in an ignorable loss rate of 1.96 $\times 10^{10} \mathrm{~cm}^{-3} \mathrm{~s}^{-1}$. Other ignorable processes include pooling reaction with the formation of the $C^{3} \Pi_{u}$ state (29) $(k=3$ $\left.\times 10^{-10} \mathrm{~cm}^{3} \mathrm{~s}^{-1}\right),{ }^{61}$ which gives a loss rate of 1.79 $\times 10^{11} \mathrm{~cm}^{-3} \mathrm{~s}^{-1}$, and radiative deexcitation (being dipole forbidden the weak Vergand-Kaplan band system has a long radiative lifetime of $\sim 2.4-3.3 \mathrm{~s}$ for $\nu=0-12 \mathrm{~s})^{7}$ which gives a loss rate of $1.28 \times 10^{10} \mathrm{~cm}^{-3} \mathrm{~s}^{-1}$. Therefore, the rate equation for the $A^{3} \Sigma_{u}^{+}$state is

$$
\begin{aligned}
d\left[\mathrm{~N}_{2}(A)\right] / d t \cong & k_{\mathrm{N}_{2}}^{X \rightarrow A} n_{e}\left[\mathrm{~N}_{2}(X)\right]-k_{\mathrm{N}_{2}}^{A \rightarrow B} n_{e}\left[\mathrm{~N}_{2}(A)\right] \\
& -k_{\mathrm{N}_{2}}^{q}\left[\mathrm{~N}\left({ }^{4} S\right)\right]\left[\mathrm{N}_{2}(A)\right]-\left[\mathrm{N}_{2}(A)\right] / \tau_{\mathrm{N}_{2}}^{D} .
\end{aligned}
$$

Solving this simplified three-state coupling kinetic model in the steady state for the $B$ state population gives 


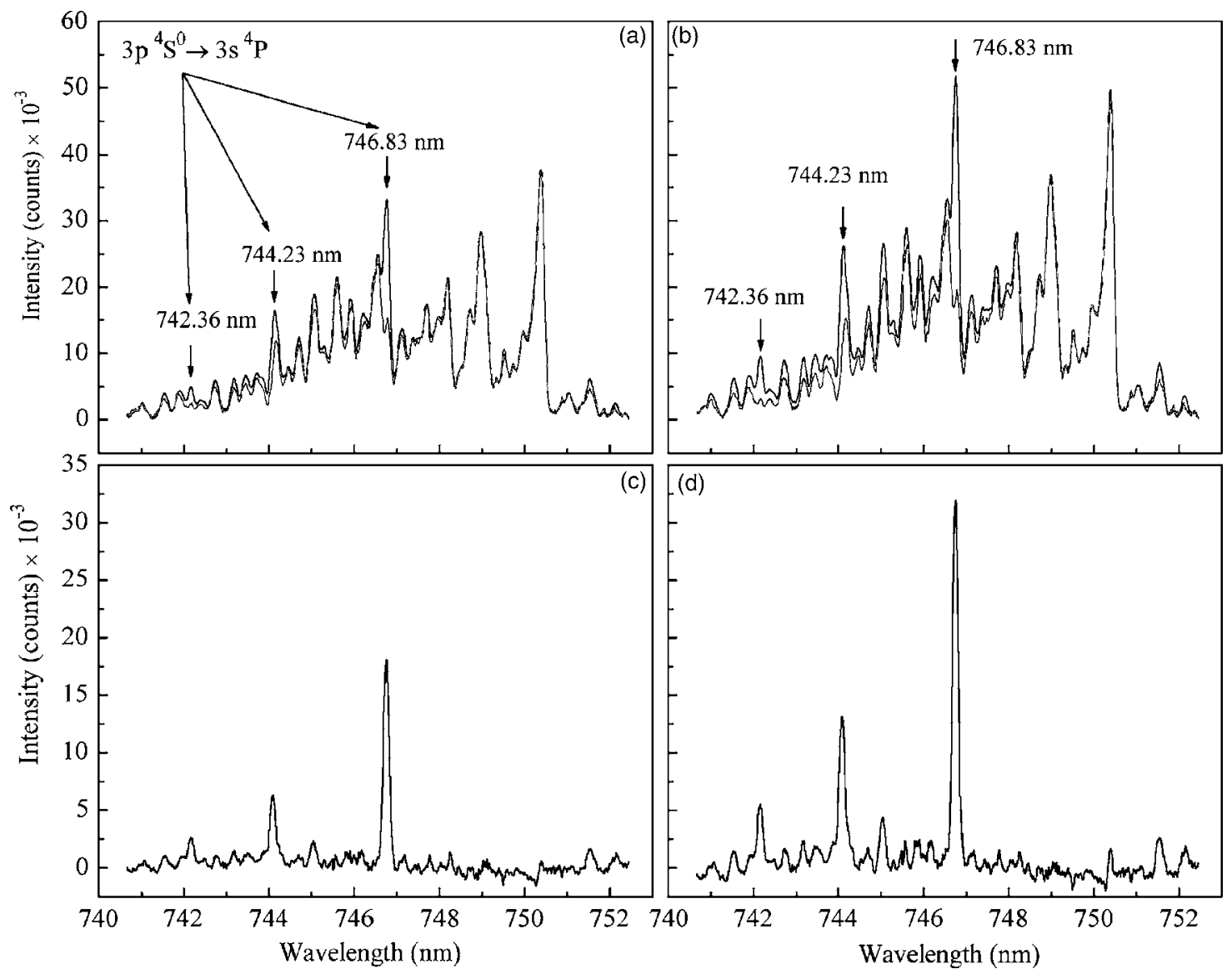

FIG. 5. Experimental 4-2 band spectra (black line) and corresponding numerically generated spectra (gray line) for (a) $E$-mode and (b) $H$-mode operating regimes; corresponding N I triplet lines obtained by difference between experimental spectra and generated spectra (c) and (d).

$$
\left[\mathrm{N}_{2}(B)\right]=K_{\mathrm{N}_{2}} n_{e}\left[\mathrm{~N}_{2}(X)\right] \tau_{\mathrm{N}_{2}}^{\mathrm{rad}},
$$

where $K_{\mathrm{N}_{2}}$ is the total rate coefficient

$$
K_{\mathrm{N}_{2}}=k_{\mathrm{N}_{2}}^{X \rightarrow B}\left\{1+\frac{k_{\mathrm{N}_{2}}^{X \rightarrow A}}{k_{\mathrm{N}_{2}}^{X \rightarrow B}} \frac{k_{\mathrm{N}_{2}}^{A \rightarrow B} n_{e} \tau_{\mathrm{N}_{2}}^{D}}{1+k_{\mathrm{N}_{2}}^{A \rightarrow B} n_{e} \tau_{\mathrm{N}_{2}}^{D}+k_{\mathrm{N}_{2}}^{q}\left[\mathrm{~N}\left({ }^{4} S\right)\right] \tau_{\mathrm{N}_{2}}^{D}}\right\} .
$$

Note that $K_{\mathrm{N}_{2}}$ depends on the atom population $\left[\mathrm{N}\left({ }^{4} S\right)\right]$, i.e., the dissociation degree.

\section{DISSOCIATION DEGREE}

The ratio of the atomic line intensity for excited atomic population $\left[N_{j}\right]$ to the $4-2$ band intensity arising from the excited $\nu^{\prime}=4$ vibrational level $\left[\mathrm{N}_{2}^{\nu^{\prime}=4}\right]$ is

$$
\frac{I_{\mathrm{N}}^{i}}{I_{\mathrm{N}_{2}}}=\frac{\lambda_{\mathrm{N}_{2}}}{\lambda_{\mathrm{N}}^{i}} \frac{S\left(\lambda_{\mathrm{N}}^{i}\right)}{S\left(\lambda_{\mathrm{N}_{2}}\right)} \frac{b_{\mathrm{N}}^{i}}{b_{\mathrm{N}_{2}}} \frac{1}{f q} \frac{K_{\mathrm{N}}}{K_{\mathrm{N}_{2}}} \frac{\tau_{\mathrm{N}}^{\mathrm{rad}}}{\tau_{\mathrm{N}_{2}}^{\mathrm{rad}}} \frac{\left[\mathrm{N}\left({ }^{4} S\right)\right]}{\left[\mathrm{N}_{2}(X)\right]},
$$

where $i$ is the index for the atomic lines of the triplet and where we have dropped indices for $f$ and $q$. Thus, by defining the dissociation degree as the ratio between the number of dissociated molecules to the initial number of molecules $\left[\mathrm{N}_{2}^{0}\right]$ and from the conservation of number of particles

$$
\left[\mathrm{N}_{2}^{0}\right] \cong\left[\mathrm{N}_{2}(X)\right]+\frac{\left[\mathrm{N}\left({ }^{4} S\right)\right]}{2},
$$

where the total number of molecules and total number of atoms are approximated by their ground state populations, the atomic line/molecular band intensity ratio can be related to the dissociation degree through

$$
\frac{I_{\mathrm{N}}^{i}}{I_{\mathrm{N}_{2}}}=\xi_{i} \frac{2 D}{1-D},
$$

where $\quad \xi_{i}=\left(\lambda_{\mathrm{N}_{2}} / \lambda_{\mathrm{N}}^{i}\right)\left(S_{\mathrm{N}}^{i} / S_{\mathrm{N}_{2}}\right)\left(b_{\mathrm{N}}^{i} / b_{\mathrm{N}_{2}}\right)\left(K_{\mathrm{N}} / K_{\mathrm{N}_{2}}\right)\left(\tau_{\mathrm{N}}^{\mathrm{rad}} / \tau_{\mathrm{N}_{2}}^{\mathrm{rad}}\right)$ $\times(1 / f q)$ is a unique parameter for each line of the triplet.

To perform our OES measurements we used the same optical chain described in detail in Part I. ${ }^{9}$ To determine the dissociation degree, first we obtained the gas temperature from the fit of the 2-0 band of the first positive system. Then, using the experimentally determined gas temperature, we synthetically generated an emission spectrum for the 4-2 band. The triplet line intensities were found by subtracting the synthetically generated spectrum from the measured 4-2 spectrum, as shown in Fig. 5. Finally, the dissociation degree was inferred from the slope of the three points obtained by calculating $I_{\mathrm{N}}^{i} / I_{\mathrm{N}_{2}}=f\left(\xi_{i}\right)$, as shown in Fig. 6 . Since $K_{\mathrm{N}_{2}}$ depends on $D$ through $\left[\mathrm{N}\left({ }^{4} S\right)\right]=2 D\left[\mathrm{~N}_{2}^{0}\right]$ an iterative procedure is employed beginning with an arbitrary initial value for $D$ $(0<D<1)$. For our plasma parameters, the correction term in Eq. (34) is relatively small $(\sim 4 \%)$ and therefore $D$ rap- 


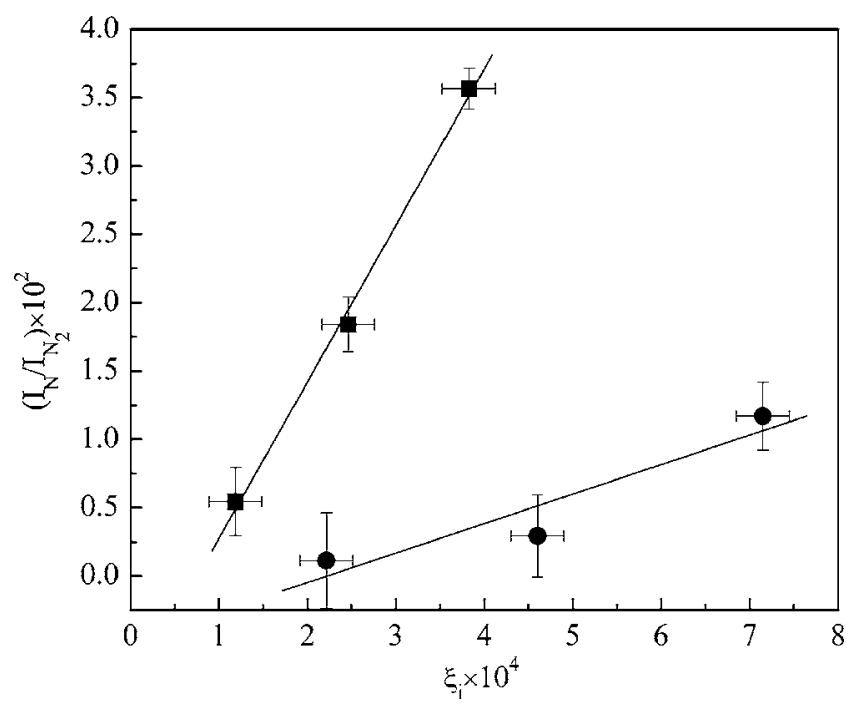

FIG. 6. Determination of the dissociation degree from the plot of atomic line/molecular band intensities ratio vs characteristic line parameter: squares, $H$-mode regime; circles, $E$-mode regime.

idly converges to its steady-state value. The results are consistent with the common assumption that excitation of the $B$ state occurs primarily through electron impact from the ground state. However, for other plasma parameters, especially higher electron densities, the correction term could significantly affect the value of the total excitation rate coefficient.

The inferred values of the dissociation degree were $5 \%$ and $13 \%$ for $E$ mode and $H$ mode, respectively. Given the characteristics of our discharge, the obtained values are reasonable. Since the measurements were performed in the expansion chamber, these low dissociation values make this plasma source a promising candidate for excited molecular species production with a direct applicability to plasma assisted molecular beam epitaxy.

\section{CONCLUDING REMARKS}

The analysis method presented here can be easily implemented for nitrogen plasma sources where continuous monitoring of the dissociation degree is desired. As shown in Fig. 4(a), the band intensities from the investigated sequences lie on the same straight line, i.e., obey a Boltzmann distribution. Therefore, to obtain the vibrational distribution necessary for finding the fraction $f$, for calculation of the weighted values of $\tau_{\mathrm{N}_{2}}^{\mathrm{rad}}$, and for determining the weighted value of $q$ from the ground state distribution, it is sufficient to only measure the $\Delta \nu=2$ sequence (see Fig. 3), i.e., the $710-780 \mathrm{~nm}$ spectral range. Then, instead of using integrated band intensities, the band head intensities can be used. Further, in calculating $D$ the 4-2 band head intensity along with the intensities of each of the atomic lines can be used. As shown in Fig. 7, after accounting for a scaling factor that relates the band head area to the total band area and is a function of gas temperature (28.4 for $H$ mode with $T_{\text {gas }}=360 \mathrm{~K}$ and 29.3 for $E$ mode with $T_{\text {gas }}=320 \mathrm{~K}$ ), the correspondence between line intensity ratios determined from the full analysis technique described here and the simple comparison of peak intensity to band

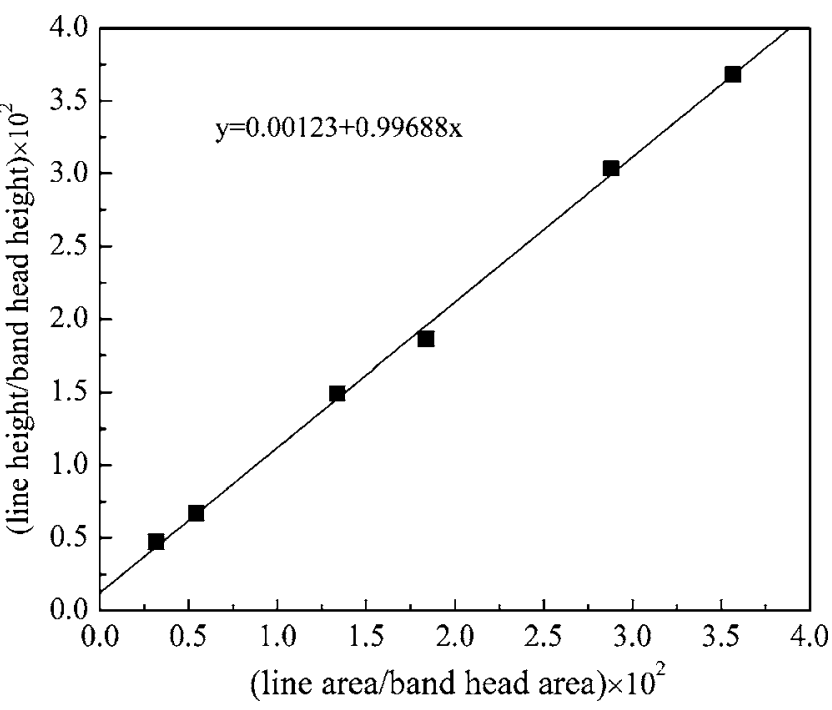

FIG. 7. Linear dependence of line/band intensities ratio obtained from lines and band head heights against the same ratio obtained from integrated intensities.

head intensity is quite good. Furthermore, since the 4-2 band spans only $10 \mathrm{~nm}$, the spectral sensitivity ratio in Eq. (35) is equal to unity (for our optical path it varied from 1.008 to 1.035 over the $10 \mathrm{~nm}$ range, thus setting it equal to one that leads to less than a $3 \%$ error in the estimated value of $D$ ). Using the triplet line intensities and the 4-2 band head heights in reports by other research groups (Fig. 3 in Ref. 12), and assuming an electron density of $10^{11} \mathrm{~cm}^{-3}$, electron temperature of 3-4 eV, and a vibrational temperature of $6000 \mathrm{~K}$, we estimate a dissociation degree of $\sim 50 \%-60 \%$ for their plasma, very close to the $\sim 70 \%$ dissociation degree value obtained through QMS and Ar actinometry measurements.

\section{ACKNOWLEDGMENTS}

One of the authors (C.B.) gratefully acknowledges Dr. Milan Simek and Dr. Santolo De Benedictis for enlightening discussions and for providing the rotational line positions for the 4-2 band.

${ }^{1}$ V. V. Uglov, V. V. Khodasevich, A. K. Kuleshov, J. A. Fedotova, D. P. Rusalsky, R. Guenzel, and E. Richter, J. Vac. Sci. Technol. B 17, 836 (1999).

${ }^{2}$ Z. X. Cao, J. Vac. Sci. Technol. A 19, 485 (2001).

${ }^{3}$ X. Tian, R. Fu, and P. Chu, J. Vac. Sci. Technol. A 20, 160 (2002).

${ }^{4}$ D. C. Jordan, C. T. Burns, and R. B. Doak, J. Appl. Phys. 89, 883 (2001).

${ }^{5}$ A. J. Ptak, M. R. Millecchia, T. H. Myers, K. S. Ziemer, and C. D. Stinespring, Appl. Phys. Lett. 74, 3836 (1999).

${ }^{6}$ T. H. Myers, M. R. Millecchia, A. J. Ptak, K. S. Ziemer, and C. D. Stinespring, J. Vac. Sci. Technol. B 17, 1654 (1999).

${ }^{7}$ L. G. Piper, J. Chem. Phys. 99, 3174 (1993).

${ }^{8}$ W. C. Hughes, W. H. Rowland, Jr., M. A. L. Johnson, S. Fujita, J. W. Cook, Jr., J. F. Schtzina, J. Ren, and J. A. Edmond, J. Vac. Sci. Technol. B 13, 1571 (1995)

${ }^{9}$ C. Biloiu, X. Sun, Z. Harvey, and E. Scime, J. Appl. Phys. 101, 073303 (2007).

${ }^{10}$ A. Lofthus and P. Krupenie, J. Phys. Chem. Ref. Data 6, 115 (1977).

${ }^{11}$ S. Agarwal, B. Hoex, M. C. M. van de Sanden, D. Maroudas, and E. Aydil, Appl. Phys. Lett. 83, 4918 (2003).

${ }^{12}$ T. Czerwiec, F. Greer, and D. B. Graves, J. Phys. D 38, 4278 (2005).

${ }^{13}$ R. P. Vaudo, J. W. Cook, Jr., and F. Schetzina, J. Vac. Sci. Technol. B 12, 1232 (1994) 
${ }^{14}$ R. W. McCullogh, J. Geddes, J. A. Croucher, J. M. Woolsey, D. P. Higgins, M. Chalp, and H. B. Gilbody, J. Vac. Sci. Technol. A 14, 152 (1996).

${ }^{15}$ D. Voulot, R. W. McCullogh, W. R. Thompson, D. Burns, J. Geddes, G. J. Cosimini, E. Nelson, P. P. Chow, and J. Klaasen, J. Vac. Sci. Technol. A 16, 3434 (1998).

${ }^{16}$ K. Sasaki, H. Kokubu, D. Hayashi, and K. Kadota, Thin Solid Films 386 243 (2001).

${ }^{17}$ N. Itagaki, S. Iwata, K. Muta, A. Yonesu, S. Kawakami, N. Ishii, and Y. Kawai, Thin Solid Films 435, 259 (2003).

${ }^{18}$ T. Nakano, S. Kumagai, and S. Samukawa, J. Appl. Phys. 92, 2990 (2002).

${ }^{19}$ H. Carrere, A. Arnoult, E. Bedel-Pereira, and A. Richard, J. Vac. Sci. Technol. B 22, 2448 (2004); J. Cryst. Growth 243, 295 (2002).

${ }^{20}$ S. Miyake, Y. Setsuhara, K. Shibata, M. Kumagai, Y. Sakawa, and T Shoji, Surf. Coat. Technol. 116-119, 11 (1999).

${ }^{21}$ M. M. Turner and M. B. Hopkins, Phys. Rev. Lett. 69, 3511 (1992).

${ }^{22}$ M. J. Druyvesteyn, Z. Phys. 64, 781 (1930).

${ }^{23}$ C. Biloiu, E. Scime, X. Sun, and B. McGeehan, Rev. Sci. Instrum. 75 , 4296 (2004).

${ }^{24}$ V. A. Godyak, R. B. Piejak, and B. M. Alexandrovich, Plasma Sources Sci. Technol. 1, 36 (1992).

${ }^{25}$ G. J. Schultz, Rev. Mod. Phys. 45, 423 (1973).

${ }^{26}$ V. M. Donnelly, J. Phys. D 37, R217 (2004).

${ }^{27}$ S. S. Tayal and O. Zatsarinny, J. Phys. B 38, 3631 (2005).

${ }^{28}$ P. S. Ganas, J. Chem. Phys. 59, 5411 (1973).

${ }^{29}$ R. M. Frost, P. Awakowicz, H. P. Summers, and N. R. Badnell, J. Appl. Phys. 84, 2989 (1998).

${ }^{30}$ P. N. Stanton and R. St. John, J. Opt. Soc. Am. 59, 252 (1969).

${ }^{31}$ Y. Itikawa, J. Phys. Chem. Ref. Data 35, 31 (2006).

${ }^{32}$ D. C. Cartwright, J. Appl. Phys. 49, 3855 (1978).

${ }^{33}$ W. L. Borst, Phys. Rev. A 5, 648 (1969).

${ }^{34}$ V. M. Huo, in Nonequilibrium Processes in Partially Ionized Gases, edited by M. Capitelli and J. N. Bardsley (Plenum, New York, 1990), Vol. B220, p. 341.

${ }^{35}$ D. C. Cartwright, S. Trajmar, A. Chutjian, and W. Williams, Phys. Rev. A 16, 1041 (1977).

${ }^{36}$ M. V. Malyshev and V. M. Donnelly, J. Vac. Sci. Technol. A 15, 550 (1997).

${ }^{37}$ M. A. Lieberman and A. J. Lichtenberg, Principles of Plasma Discharges and Material Processing (Wiley, New York, 1994), p. 257.
${ }^{38}$ J. Musielok, W. L. Wiese, and G. Veres, Phys. Rev. A 51, 3588 (1995).

${ }^{39}$ NIST Atomic Spectra Database, www.nist.gov

${ }^{40}$ J. Levanton, A. Richard, J. Henriques, H. R. T. Silva, and J. Amorim, J. Phys. D 39, 3285 (2006).

${ }^{41}$ A. Catherinot and A. Sy, Phys. Rev. A 20, 1511 (1979).

${ }^{42}$ L. G. Piper, K. W. Holtzclaw, B. D. Green, and W. A. M. Blumberg, J. Chem. Phys. 90, 5337 (1989).

${ }^{43}$ C. O. Laux and C. H. Kruger, J. Quant. Spectrosc. Radiat. Transf. 48, 9 (1992).

${ }^{44}$ F. R. Gilmore, R. R. Laher, P. J. Espy, and C. H. Kruger, J. Phys. Chem. Ref. Data 21, 1005 (1992)

${ }^{45}$ G. Cernogora, C. M. Ferreira, L. Houchard, M. Touzeau, and J. Loureiro, J. Phys. B 17, 4429 (1984).

${ }^{46}$ J. Levanton, J. Amorim, A. R. Souza, D. Franco, and A. Richard, J. Phys. D 35, 689 (2002).

${ }^{47}$ C. Biloiu, X. Sun, Z. Harvey, and E. Scime, Rev. Sci. Instrum. 77, 10F117 (2006).

${ }^{48}$ H. Coitout and G. Cernogora, J. Phys. D 39, 1821 (2006).

${ }^{49}$ N. Britum, M. Gillard, A. Richard, Y. M. Kim, K. S. Kim, and J. G. Han, J. Phys. D 40, 1022 (2007).

${ }^{50}$ B. Krames, Th. G.-Meyer, and J. Meichsner, J. Appl. Phys. 89, 3115 (2001).

${ }^{51}$ V. Guerra and J. Loureiro, Plasma Sources Sci. Technol. 6, 361 (1997).

${ }^{52}$ B. Gordiets, C. Ferreira, V. Guerra, J. Loureiro, J. Nahorny, D. Pagnon, M. Touzeau, and M. Vialle, IEEE Trans. Plasma Sci. 23, 750 (1995).

${ }^{53}$ L. G. Piper, J. Chem. Phys. 90, 5337 (1989).

${ }^{54}$ H. Geisen, D. Neuschafer, and Ch. Ottinger, J. Chem. Phys. 92, 104 (1990).

${ }^{55}$ S. DeBenedictis, G. Dilecce, and M. Simek, J. Chem. Phys. 110, 2947 (1999).

${ }^{56}$ G. Cernogora, L. Houchard, M. Touzeau, and C. M. Ferreira, J. Phys. B 14, 2977 (1981)

${ }^{57}$ L. G. Piper, J. Chem. Phys. 90, 7087 (1989).

${ }^{58}$ C. Foissac, A. Camparague, A. Kachanov, P. Supiot, G. Weirauch, and N. Sadeghi, J. Phys. D 33, 2434 (2000).

${ }^{59}$ D. Levron and A. V. Phelps, J. Chem. Phys. 69, 2260 (1978).

${ }^{60}$ W. G. Clark and D. W. Setser, J. Phys. Chem. 84, 2226 (1980).

${ }^{61}$ L. G. Piper, J. Chem. Phys. 88, 6911 (1988). 